\title{
SIGOA-DF: Plataforma Para Inspeção e Gestão de Obras de Arte Especiais no Distrito Federal Utilizando a Metodologia do GDE/UnB
}

\author{
G. Berbert-Born ${ }^{1 *}$, A. Nascimento Filho ${ }^{1}$, J.G. Monteiro ${ }^{1}$, M. E. Pereira ${ }^{1}$, M. H. Oliveira ${ }^{1}$, C. \\ Saraiva \\ *Autor de Contato: guiborn.eng@gmail.com \\ ${ }^{1}$ Departamento de Engenharia Civil e Ambiental, Faculdade de Tecnologia, Universidade de Brasília, Brasil
}

\begin{abstract}
RESUMO
Um sistema de gerenciamento de Obras de Artes especiais é essencial para o mapeamento de pontes e viadutos em nível crítico de deterioração e, consequentemente, o auxílio na tomada de decisões para novas manutenções. Este trabalho objetiva o desenvolvimento de um sistema de gerenciamento que seja eficiente e confiável para o controle de Obras de Arte Especiais, utilizando a metodologia do grau de deterioração desenvolvida pela Universidade de Brasília, o SIGOA-DF. O desenvolvimento do sistema contempla um módulo de inspeções para obtenção dos dados, o ajuste da ferramenta conforme às necessidades dos inspetores e um módulo análise dos resultados obtidos e armazenados no banco de dados.
\end{abstract}

Palavras Chaves: Sistema de Gerenciamento; Pontes e viadutos; Manutenções; Grau de deterioração. 


\begin{abstract}
A management system for bridges and viaducts is essential for mapping structures in a critical level of deterioration and consequently to support decisions for maintenance processes. This work objectives the development of a management system that is efficient and reliable for the knowledge and control of the deterioration processes in structures, using the methodology of the degree of deterioration developed at the University of Brasilia, the SIGOA-DF. The development of the system includes a inspection module to obtain data, adjusting the tool according to the needs of inspectors and a module of analysis for the results obtained and stored in the database.
\end{abstract}

Keywords: Management System; Bridges and viaducts; Maintenance; Degree of deterioration.

\title{
RESUMEN
}

Un sistema de gestión de obras de artes especiales es fundamental para el mapeo de puentes y viaductos en un nivel crítico de deterioro y, en consecuencia, la ayuda en la toma de decisiones de nuevo mantenimiento. Este trabajo tiene como objetivo desarrollar un sistema de gestión eficiente y confiable para el control de Obras de Arte Especiales, utilizando la metodología del grado de deterioro desarrollada en la Universidad de Brasilia, el SIGOA-DF. El desarrollo del sistema incluye un módulo de inspección para obtener los datos, ajustamiento de la herramienta según las necesidades de los inspectores y un módulo para analizar los resultados obtenidos y almacenados en la base de datos.

Palabras clave: Sistema de Gestión; Puentes y viaductos; Mantenimiento; Grado de deterioro.

\section{INTRODUÇÃO}

As Obras de Arte Especiais (OAEs) são estruturas que têm a função de ligar pontos separados por obstáculos naturais ou artificiais. Essas construções estão submetidas às ações externas provenientes de cargas móveis de veículos, forças devido ao vento, exposição à água, variações de temperatura, retração e fluência no caso do concreto. As OAEs são afetadas pelo envelhecimento, fadiga, corrosão, ausência ou falha de manutenção, pelo incremento de cargas, entre outros fatores. Em relação às estruturas de concreto armado, devem ser atendidos os requisitos de qualidade, classificados em capacidade resistente, desempenho em serviço e durabilidade. Nota-se que a capacidade resistente está relacionada à segurança à ruptura da estrutura. A capacidade da estrutura atender as condições de utilização durante sua vida útil evidencia o desempenho em serviço. Enquanto a durabilidade está relacionada ao grau de exposição da estrutura à fatores ambientais ao longo do tempo e à boa conduta de utilização dos usuários.

Conforme evidenciado por Mitre (2005), é fundamental observar que a maioria dos processos de deterioração desenvolve-se gradualmente e tem manifestações visíveis ou detectáveis por ensaios específicos. Dessa forma, pode-se identificar, diagnosticar e solucionar os problemas antes que atinjam proporções graves ou que resultem em custos financeiros e sociais elevados de recuperação. Por essa ótica, o acompanhamento periódico do estado das OAEs, por meio de vistorias e inspeções detalhadas, executadas conforme um conjunto de procedimentos padronizados e tecnicamente adequados, é o modo mais eficaz de subsidiar uma política de manutenção corretiva e preventiva de OAEs.

Inspeções detalhadas permitem identificar as principais manifestações patológicas atuantes nas estruturas em análise. Caso seja necessário, é possível promover planos de manutenção, a fim de 
que se possa atender os requisitos mínimos de capacidade de carga, segurança e conforto, permitindo a ampliação do período de preservação e de serviço das edificações. A avaliação visual pode ser utilizada como método de inspeção, bem como a realização de ensaios não destrutivos dos elementos das OAEs.

A metodologia do Grau de Deterioração Estrutural (GDE/UnB) trata-se de um método que faz uso de equações para análise do grau de deterioração estrutural, além de permitir a avaliação, separadamente, de cada elemento da estrutura. Essa metodologia pode ser utilizada como uma ferramenta de apoio à decisão dos gestores, permitindo identificar as principais manifestações patológicas que afetam as estruturas e, se necessário, promover um plano de manutenção, a fim de que possam atender os requisitos mínimos de capacidade de carga, segurança, conforto e assim ampliar seu período de preservação e serviço.

Desenvolvida por Castro (1994), para quantificar o grau de deterioração em estruturas de concreto armado convencionais, a metodologia evoluiu por meio das pesquisas de Lopes (1998), Boldo (2002), Fonseca (2007), Euqueres (2011) e Verly (2015). As adaptações evidenciadas por esses autores incidem nas formulações e parâmetros utilizados para o desenvolvimento da plataforma para inspeção e gestão de obras de arte especiais no Distrito Federal, o SIGOA-DF, exposto no presente trabalho.

De acordo com a metodologia, as edificações inspecionadas podem ser particionadas, tomando como medida de separação as características estruturais dos elementos, em grupos denominados famílias de elementos. Cada família detém uma matriz com possíveis manifestações de danos. Cada possibilidade de manifestação acompanha um valor de Fator de Ponderação do Dano $\left(\mathrm{F}_{\mathrm{p}}\right)$ com o objetivo de quantificar a relevância de um determinado dano em função dos quesitos de estética, funcionalidade e segurança. Com a inspeção visual, o responsável técnico deve atribuir o Fator de Intensidade do Dano $\left(\mathrm{F}_{\mathrm{i}}\right)$, classificando assim a gravidade do dano. A fase seguinte da metodologia diz respeito ao Grau do Dano $(D)$, fator introduzido na metodologia para quantificar a manifestação de cada dano no elemento com base na evolução temporal da deterioração do concreto. Por meio desses dados, é possível calcular o Grau de Deterioração de um Elemento $\left(\mathrm{G}_{\mathrm{de}}\right)$ e, em sequência, determinar o Grau de Deterioração de uma Família de Elementos $\left(\mathrm{G}_{\mathrm{df}}\right)$ dado pela média aritmética entre os graus de deterioração dos elementos componentes da família.

Conforme indicado por Castro (1994), cada família de elementos em análise deve possuir um fator de relevância estrutural $\left(\mathrm{F}_{\mathrm{r}}\right)$, de modo a considerar a importância relativa das diversas famílias de elementos no conjunto de partições da obra. O parâmetro final de análise é definido como o Grau de Deterioração da Estrutura $\left(\mathrm{G}_{\mathrm{d}}\right)$ composto pela média ponderada dos graus de deterioração das famílias de elementos e seus respectivos fatores de relevância estrutural. Com a obtenção desse valor, é possível classificar a estrutura analisada em níveis que variam de estado baixo a crítico de deterioração, de acordo com a separação feita por Euqueres (2011). A Figura 1 mostra o fluxograma adaptado de Castro (1994) proposto para a metodologia GDE/UnB e suas etapas de avaliação.

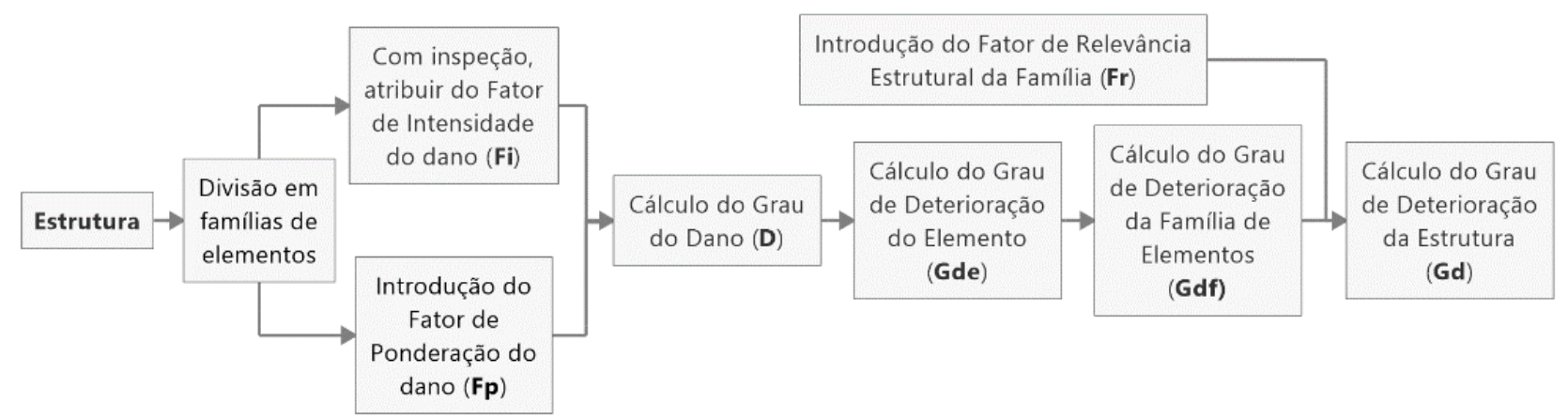

Figura 1 - Fluxograma da metodologia GDE/UnB (CASTRO,1994 - adaptado) 
As Obras de Arte Especiais são de grande importância para o desenvolvimento socioeconômico do país, sendo a manutenção algo fundamental para a prolongação e aproveitamento máximo da vida útil das estruturas. Para tal, é essencial que o sistema de gerenciamento que seja eficiente e confiável para o controle das OAEs, especialmente para o mapeamento das estruturas em estado crítico e o auxílio na tomada de decisões a serem realizadas.

Para garantir maior controle das pontes e viadutos sob sua responsabilidade, uma entidade deve idealmente utilizar um sistema de gerenciamento de OAEs, no qual seja possível a consulta de um histórico de inspeções, as quais devem preferencialmente ser detalhadas. A depender da quantidade de OAEs no banco de dados, torna-se difícil a consulta individual das mesmas, sendo a melhor forma de controle a criação de indicadores e parâmetros quantitativos que permitam a caracterização do estado de conservação, além do histórico de inspeções e manutenção de diversas estruturas.

Elbehairy (2007), afirma que um sistema de gerenciamento de OAEs pode ser definido como uma ferramenta de auxílio aos gestores e entidades responsáveis para a otimização de políticas de decisão de uma rede viária, facilitando a criação de planejamento de ações de manutenção, reabilitação ou substituição, considerando-se os objetivos a longo prazo e os recursos disponíveis. De acordo com Moscoso (2017), de maneira geral, um sistema de gerenciamento de OAEs se divide em três grupos: banco de dados, análise dos dados e suporte à decisão. Segundo a autora, as decisões tomadas pelos responsáveis são baseadas nas informações de entrada dos trabalhos realizados nas OAEs e das inspeções programadas nas estruturas.

Dada a importância de um sistema de gerenciamento de OAEs, o Sistema de Gerenciamento de Obras de Arte do Distrito Federal (SIGOA-DF) foi desenvolvido como uma ferramenta de apoio à inspeção, por meio da metodologia GDE/UnB, das OAEs dessa região e, por conseguinte, auxiliar na definição de prioridades de intervenção nos elementos componentes das edificações presentes na plataforma. $\mathrm{O}$ uso desta ferramenta permite à equipe envolvida na gestão de obras de arte formular decisões consistentes e estruturadas, visando à otimização dos recursos disponíveis.

\section{PROCEDIMENTO}

Este trabalho consiste no desenvolvimento de um sistema de gerenciamento de Obras de Arte Especiais utilizando a metodologia GDE/UnB, o SIGOA-DF. Para tal, foi utilizada a linguagem Visual Basic for Applications (VBA), baseada no software Microsoft Excel, cujo uso é familiar para grande parte dos alunos de graduação, pós-graduação e no meio empresarial e governamental em geral. Esta seção aborda a estrutura do sistema desenvolvido, bem como apresenta de forma geral suas etapas de pré-processamento, processamento e pós processamento.

A plataforma SIGOA-DF vem sendo utilizada para a inspeção de diversos viadutos do Distrito Federal, sendo feitas diversas reuniões para que a mesma se ajustasse às necessidades dos inspetores. As inspeções realizadas e as Obras de Arte Especiais cadastradas na plataforma fazem, dessa forma, parte do banco de dados alimentado ao longo dos anos de 2020 e 2021.

\subsection{A utilização do VBA no desenvolvimento da plataforma}

A escolha do VBA para a implementação do SIGOA-DF se deu pela sua simplicidade de implementação e pela facilidade e familiaridade de grande parte da comunidade acadêmica e do mercado de trabalho com o Excel, o software de planilhas eletrônicas mais popular do mundo. Trata-se de uma plataforma consolidada, robusta, com alto poder de processamento e de interface amigável ao usuário. 
O VBA permite ainda acesso e manutenção de arquivos e diretório do sistema operacional do computador, que por sua vez pode ser conectado à nuvem, possibilitando a criação de um ecossistema integrado entre interface e banco de dados.

\subsection{A estrutura do SIGOA-DF}

O desenvolvimento de uma plataforma prática e intuitiva ao usuário é essencial para a boa qualidade dos dados coletados, assim como facilidade de acesso aos mesmos e aos resultados de seu processamento. Visando a organização, facilidade de uso e usabilidade, a organização do SIGOA-DF é dividida entre dois elementos principais: a interface e o banco de dados.

\subsubsection{Banco de dados}

$\mathrm{O}$ banco de dados consiste em um conjunto de informações armazenadas em nuvem, que podem ser acessadas quando a plataforma de sincronização é acessada pelo computador, sendo possível a sincronização completa ou seletiva de diretórios, além da consulta de objetos pontuais, como fichas de inspeção e fotos específicas de determinada OAE. Dessa forma, não há necessidade de armazenamento de todos os arquivos de inspeção no computador, economizando-se espaço local. Quando uma OAE é designada a um inspetor, a plataforma cria automaticamente a estrutura de diretórios, podendo ser determinadas as permissões de acesso ao inspetor A estrutura conta com diretórios, é gerado automaticamente um link para um ponto em plataforma de mapas online, podendo ser acessado em dispositivos móveis para facilitar a localização da mesma. Além disso, é gerada uma ficha de inspeção, baseada em um modelo pré-definido, que será preenchida pelo inspetor e também mostrada adiante.

Foi utilizada ainda uma Interface de Programação de Aplicativos (API) que permite que sejam obtidas automaticamente imagens da localização da OAE no mapa, bem como imagens de satélite e imagens a nível de terreno de serviços de terceiros, quando disponíveis no local.

Por fim, é criada automaticamente um diretório no qual deverá ser inserido o registro fotográfico feito pelo inspetor. Este deve ser organizado por elemento, para que seja possível a realização de eventuais consultas dentro do banco de dados. A Figura 2 mostra a estrutura utilizada no banco de dados do SIGOA-DF.

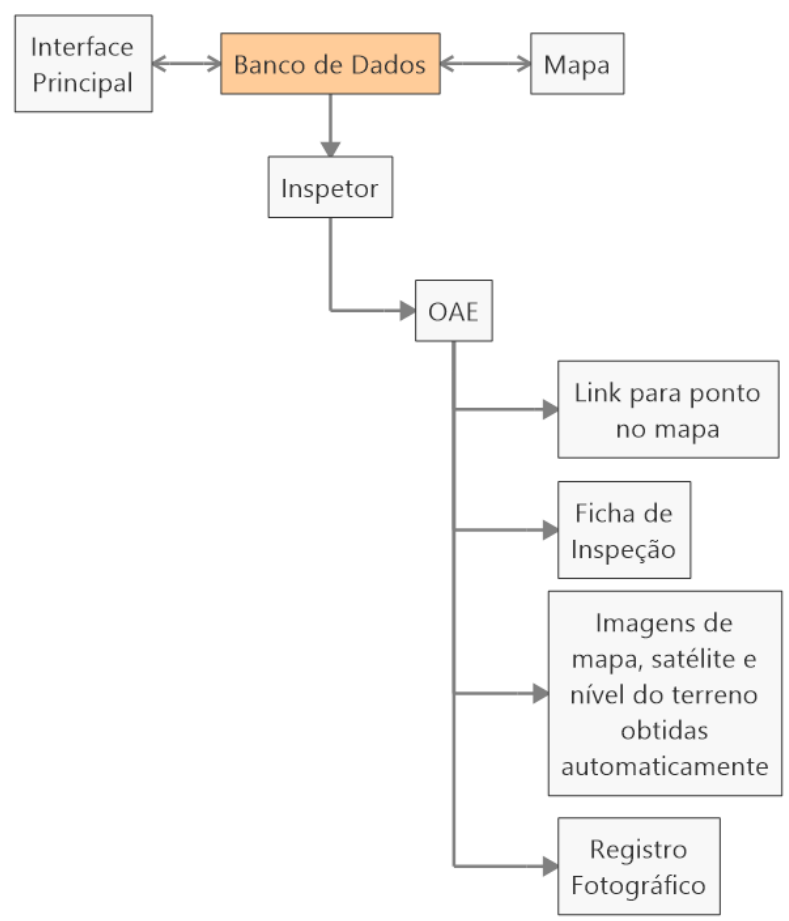

Figura 2 - Estrutura do Banco de Dados do SIGOA-DF 


\subsubsection{Integração a ferramentas de georreferenciamento}

O banco de dados está ligado à Interface Principal, que gerencia as OAEs cadastradas e é capaz de importar e exportar arquivos de extensão KML e KMZ. Com isso, as informações básicas de uma OAE podem ser acessadas por softwares de georreferenciamento. Algumas dessas plataformas possibilitam a categorização dos pontos no mapa de acordo com informações tais como inspetor responsável, grau de dano e estado de conservação, conforme mostrado na Figura 3.

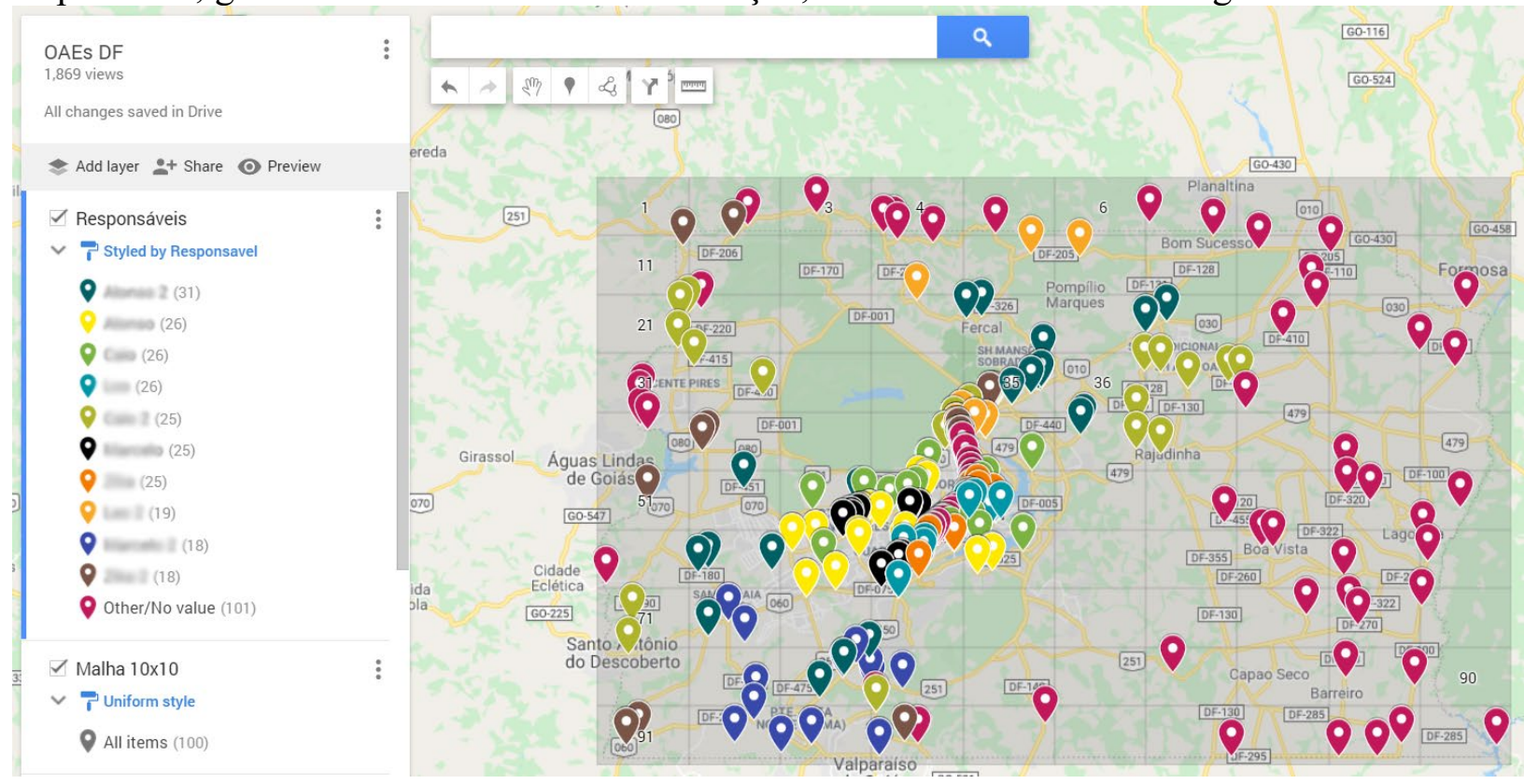

Figura 3 - Interface de mapa colaborativo com classificação por inspetor

A utilização de uma interface colaborativa permite que os inspetores tenham acesso à localização e às informações básicas das OAEs, podendo ser solicitada a adição e/ou a remoção de pontos no mapa. A localização pode ser acessada pelo GPS do celular do inspetor, facilitando o deslocamento até a estrutura.

\subsubsection{Interface Principal}

A interface é o espaço onde ocorrem as etapas de pré-processamento, processamento e pósprocessamento dos dados contidos no banco de dados. Consiste no principal ponto de contato do usuário com a plataforma, onde este pode consultar e inserir informações, além de acessar ferramentas de análise dos dados cadastrados. A interface pode ser subdividida em dois componentes: a Interface Principal e a Interface Secundária.

A Interface Principal da plataforma consiste na área de interação do usuário com o banco de dados. Nela, pode ser consultado um resumo das OAEs que compõem o banco, bem como feito o gerenciamento dessas. O esquema da Interface Principal é apresentado na Figura 4, juntamente ao menu da aba principal da ferramenta. 


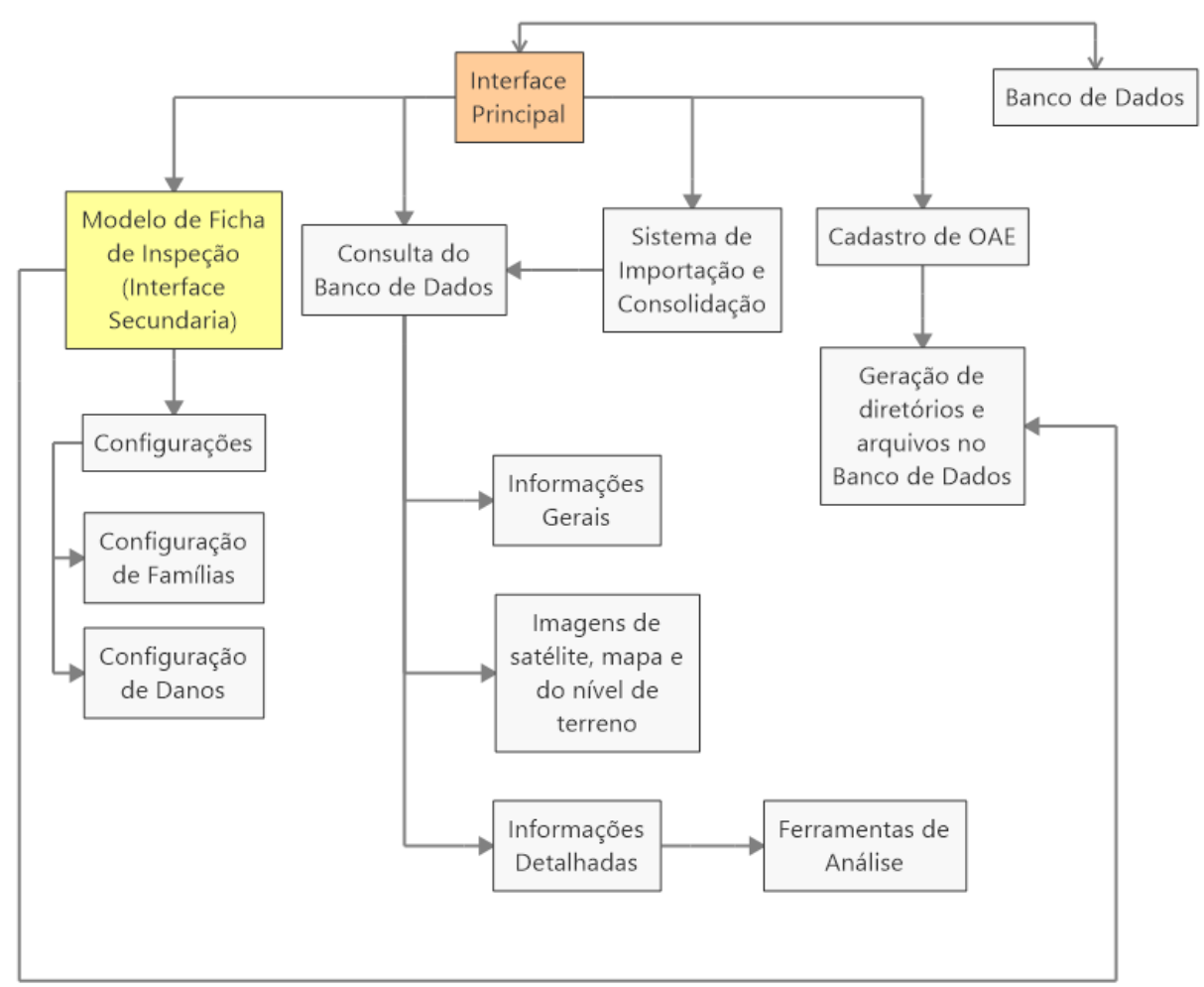

Consulta

Informações

Básicas

Criar Banco de

Dados na Nuvem

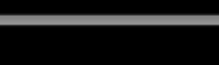

Importar KML

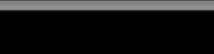

Exportar KML

Consulta

Detalhada

\section{Importar Banco}

de Dados da

Nuvem

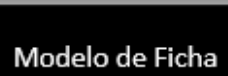

de Inspeção

Figura 4 - Esquema da Interface Principal da Plataforma (esquerda) e Menu da Aba Principal (direita)

A Interface Principal da plataforma pode ser subdividida em quatro principais funcionalidades por ela oferecidas:

a) Modelo de Ficha de Inspeção: utilizado para a reprodução das Fichas de Inspeção a serem preenchidas pelos responsáveis por cada OAE. Este modelo consiste em uma planilha a parte, que é reproduzida com os dados de cada OAE cadastrada e inserida no banco de dados. A Ficha de Inspeção consiste na Interface Secundária, que será detalhada adiante.

b) Módulo de Consulta e Análise: composto por quatro subelementos, sendo eles uma aba com informações gerais, uma aba de consulta, uma aba com informações detalhadas e uma aba de análise dos dados detalhados, a partir da qual são obtidos os resultados apresentados neste trabalho. A aba de informações gerais consiste em um resumo das informações do banco de dados, onde podem ser consultados nome, descrição, latitude, longitude, malha, observações, responsável, além dos $G_{d}$ e $G_{d}$ * calculados. Os dados dessa aba podem ser importados do arquivo KML obtido na plataforma de georreferenciamento. A partir dessas informações também podem ser gerados os diretórios e arquivos no banco de dados armazenado em nuvem, fazendo-se a filtragem da lista conforme a necessidade.

A aba de consulta, nomeada como "Principal" consiste em uma aba onde o usuário pode acessar as informações básicas das OAEs cadastradas no banco de dados, sendo possível a consulta das seguintes informações: Nome, Descrição, Tipo, Estado de Conservação, Latitude, Longitude, Malha, OBS, Responsável, Gd e Gd* (grau de dano considerando apenas elementos de $\mathrm{G}_{\mathrm{de}}>15$ ). A aba de consulta também permite a obtenção das imagens de satélite, posição no mapa e quatro vistas do nível de terreno. A seleção da OAE para a qual se deseja obter informações é feita por meio de um formulário de usuário, exposto na Figura 5 que possui opções de filtro por malhas, 
responsáveis, tipo de estrutura, estado de conservação, responsável e observação, facilitando e agilizando eventuais consultas.
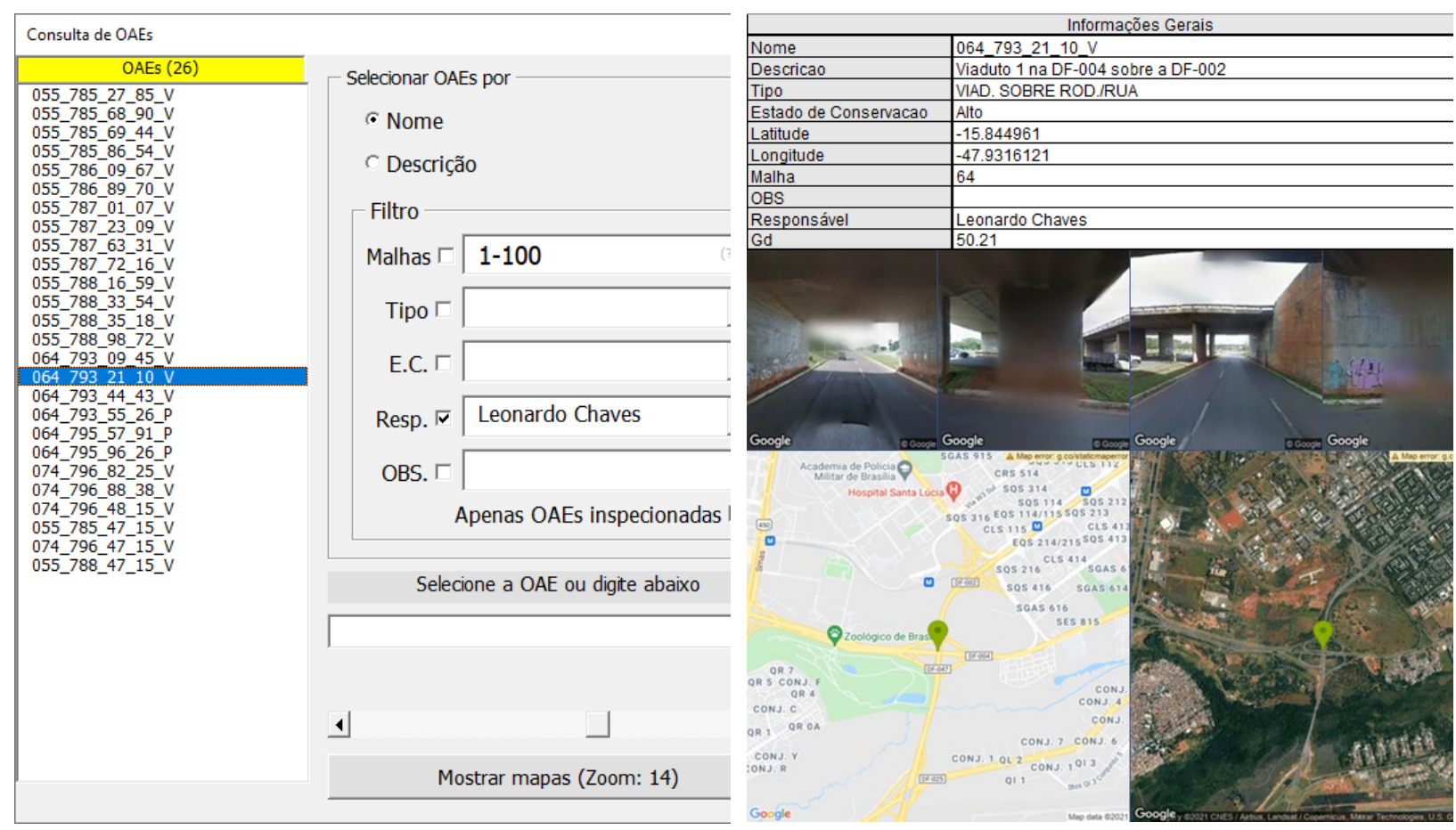

Figura 5- Consulta de Informações Básicas (esquerda) e resultados da busca (direita)

No caso da necessidade de uma consulta detalhada, o usuário pode acessar a aba "Consulta Detalhada", na qual tem acesso a uma ferramenta de análise básica a partir da qual é possível ver informações a níveis de dano, elementos e famílias para cada OAE inspecionada. A aba "Análise Geral" fornece uma análise estatística básica dos dados coletados, que será apresentada nos resultados deste trabalho.

c) Módulo de Importação e Consolidação: Este módulo concede ao usuário acesso ao ambiente no qual é capaz de importar os dados detalhados do banco de dados.

\subsubsection{Interface Secundária - Fichas de Inspeção}

A Interface Secundária consiste em fichas de inspeção geradas a partir do Modelo de Ficha de Inspeção, modelo no qual podem ser inseridas as famílias e danos padrão, bem como o layout de modelagem da OAE a ser inspecionada. Nessa interface, o inspetor deve cadastrar os elementos que compõem a OAE para a realização da inspeção. $\mathrm{O}$ usuário pode também editar os valores padrão como o Fator de Relevância $\left(F_{r}\right)$ de cada elemento, bem como os tipos e Fatores de Ponderação $\left(\mathrm{F}_{\mathrm{p}}\right)$ dos danos.

Ao ser gerado o banco de dados das OAEs a partir da Interface Principal, cria-se uma cópia do Modelo de Ficha de Inspeção, o qual é disposto dentro do diretório criado para a OAE no banco. $\mathrm{O}$ esquema de funcionamento da Interface Secundária está resumido no diagrama apresentado na Figura 6. 


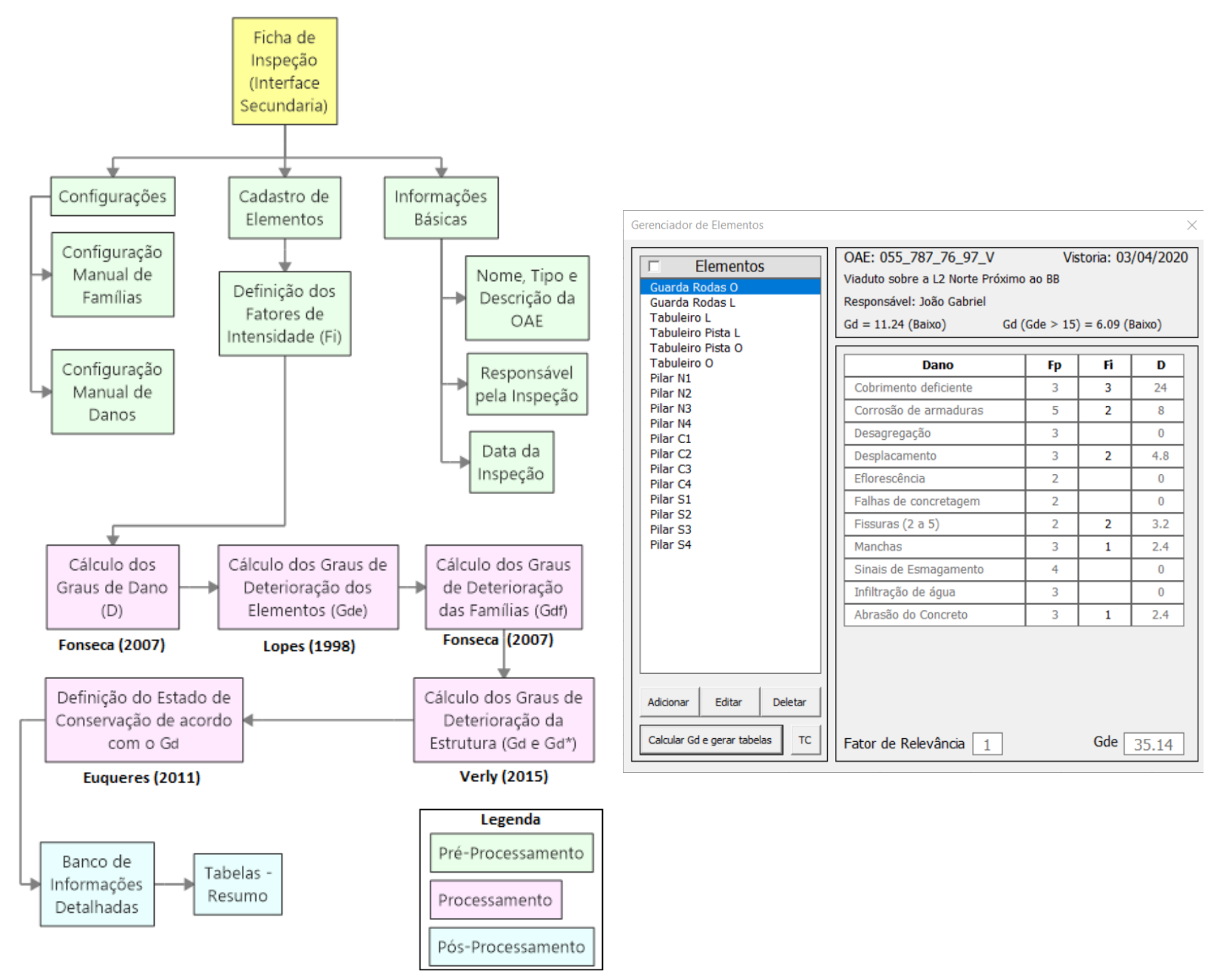

Figura 6 - Esquema representativo do funcionamento da Interface Secundária (esquerda) e Gerenciador de Elementos (direita).

A Interface Secundária permite a criação e edição de elementos dentro da OAE, sendo possível a escolha de uma família, ou tipo de elemento pré-definido, a edição ou a criação de uma nova família. Pode ainda ser gerada uma tabela para preenchimento em campo considerando os elementos cadastrados e seus respectivos danos. O intuito dessa tabela é facilitar a percepção dos pesos de cada tipo de elemento e de cada tipo de dano na estrutura global, sendo facilitado o preenchimento posterior da Ficha de Inspeção a partir da tabela impressa levada a campo. O funcionamento da Interface Secundária pode ser divido em três etapas principais: préprocessamento, processamento e pós-processamento.

a) Etapa de Pré-Processamento: consiste na inserção de informações básicas, no cadastro de elementos e na configuração das famílias e danos, quando necessário. Quando cada elemento criado é selecionado na lista de elementos, surge uma nova lista contendo os danos a ele associados, bem como seus Fatores de Ponderação $\left(F_{p}\right)$, e o Fator de Relevância $\left(F_{r}\right)$ do elemento.

b) Etapa de Processamento: à medida que são preenchidos os Fatores de Intensidade $\left(\mathrm{F}_{\mathrm{i}}\right)$ associados a um dano de um elemento, ocorre o processamento desse elemento, sendo calculado o Grau de Dano (D) e o Grau de Deterioração do Elemento $\left(\mathrm{G}_{\mathrm{de}}\right)$. Concluído o preenchimento dos 
dados para todos os elementos, o responsável pode pressionar o botão de cálculo para o processamento global da estrutura.

Para a etapa processamento, foi utilizada a metodologia GDE/UnB nas suas últimas versões e adaptações da literatura técnica, levando-se em consideração o Manual de Aplicação da Metodologia GDE/UnB a Obras de Arte Especiais proposto por Verly (2015).

Em resumo, os cálculos foram feitos definindo-se primeiramente o grau de cada dano em cada elemento cadastrado. Em seguida, o grau de deterioração do elemento é calculado. A partir dos fatores de relevância das famílias, o cálculo prossegue para o grau de deterioração da família e, em seguida, para o grau de deterioração estrutural da obra analisada.

c) Etapa de Pós-Processamento: é feita a atualização dos valores de $\mathrm{G}_{\mathrm{d}}$ e de $\mathrm{G}_{\mathrm{d}}$ * dentro do Gerenciador de Elementos. Além disso, a Interface Secundária fornece três tabelas.

A primeira tabela consiste em um resumo de cada elemento criado na estrutura, seus Fatores de Relevância (Fr), seus danos associados, os Fatores de Ponderação (Fp), os Fatores de Intensidade (Fi), bem como os Graus de Dano (D) de cada um deles. Além disso é apresentado o Grau de Deterioração de cada elemento (Gde).

A segunda tabela fornece uma lista de elementos relacionados a seus Graus de Deterioração (Gde). A partir das famílias em que cada elemento está inserido, a tabela fornece ainda o Grau de Deterioração da Familia (Gdf) e o Fator de Relevância (Fr) a elas associado. Por fim, é exposto o Grau de Deterioração Esturural (Gd) e o Nível de Deterioração equivalente da Estrutura, conforme proposto por Euqueres (2011).

A terceira tabela fornece informações semelhantes às fornecidas na segunda tabela, sendo, porém, consideradas apenas os valores de Gde maiores que 15.

Por fim, a partir dos dados obtidos no processamento, a Interface Secundária deixa preparados os dados detalhados para a importação pela Interface Principal. Com isso, padroniza-se o procedimento de coleta de dados para o sistema de análise das informações detalhadas.

\section{RESULTADOS}

Nos anos de 2020 e 2021, a plataforma desenvolvida foi utilizada por alunos da Universidade de Brasília para a inspeção de Obras de Arte Especiais do Distrito Federal. Os trabalhos de Saraiva (2020), Chaves (2020) e Pereira (2020), bem como inspeções feitas por outros alunos da universidade, consistiram na inspeção de diversas OAEs, sendo as mesmas feitas utilizando-se o SIGOA-DF. O trabalho desses alunos possibilitou o aprimoramento da ferramenta criada, tendo sido levadas em consideração sugestões de melhoria e as necessidades dos responsáveis pelas inspeções.

Foram coletados dados referentes a 168 OAEs, sendo criado um banco de informações detalhadas com aproximadamente 25000 linhas de dados. Esta seção apresenta uma análise básica dos dados preliminares coletados por meio das Fichas de Inspeção. Ressalta-se que todos os gráficos e tabelas apresentados nesse capítulo são produtos do processamento na aba "Análise Geral" da Interface Principal do SIGOA-DF.

\subsection{Análise geral das OAEs inspecionadas}

Do total de 356 viadutos e pontes levantados no Distrito Federal, 168 tiveram suas inspeções realizadas utilizando-se o SIGOA-DF nos anos de 2020 e 2021. Um panorama geral das inspeções é apresentado na Tabela 1, na qual observa-se que os valores de Grau de Deterioração (Gd) variaram em um intervalo de 0.91 (nível baixo) a 150.84 (nível crítico). O valor médio encontrado foi de 29.33, representando um nível de deterioração médio pelos critérios estabelecidos por Euqueres (2011). 
Tabela 1 - Panorama geral das OAEs levantadas e inspecionadas

\begin{tabular}{|c|c|}
\hline OAEs no Banco De Dados & 356 \\
\hline OAEs Inspecionadas & 168 \\
\hline \% Inspecionadas & $47.2 \%$ \\
\hline Gd Máximo & 150.84 \\
\hline Gd Mínimo & 0.91 \\
\hline Gd Médio & 29.33 \\
\hline
\end{tabular}

Ao analisar-se as 10 OAEs mais críticas, expostas na Tabela 2, são observados 9 viadutos e apenas uma ponte. Pode ainda ser constatado uma grande variação nos valores de $\mathrm{G}_{\mathrm{d}}$ encontrados, sendo 150.84 o maior deles (crítico) e 82.98 o menor (sofrível). A estrutura mais crítica apresenta, portanto, um Grau de Deterioração aproximadamente $82 \%$ maior que a décima estrutura mais crítica do banco de dados.

Analisando-se as 10 OAEs menos críticas, também expostas na Tabela 2, são observados apenas viadutos. Neste caso, constata-se menor variação nos valores de $\mathrm{G}_{\mathrm{d}}$ encontrados, sendo $4.10 \mathrm{o}$ maior deles (baixo) e 0.91 o menor (baixo). A décima estrutura menos crítica apresenta, dessa forma, um Grau de Deterioração aproximadamente 351\% maior que a estrutura menos crítica do banco de dados.

Tabela 2 - Relação das 10 OAEs mais críticas e das 10 OAEs menos críticas

\begin{tabular}{|c|c|c|c|}
\hline \multicolumn{2}{|c|}{ OAEs mais críticas } & \multicolumn{2}{|c|}{ OAEs menos críticas } \\
\hline OAE & Gd & OAE & Gd \\
\hline $\begin{array}{lllll}064 & 789 & 22 & 89 & \mathrm{~V}\end{array}$ & 150.84 & $\begin{array}{lllll}044 & 789 & 89 & 51 & \mathrm{~V}\end{array}$ & 4.10 \\
\hline $064 \quad 795 \quad 85 \quad 47 \mathrm{~V}$ & 142.84 & $055 \quad 788 \quad 44 \quad 84 \quad \mathrm{~V}$ & 4.08 \\
\hline $064 \_795 \_54 \_28 \_\mathrm{P}$ & 133.20 & $044 \quad 789 \_68 \_00 \_\mathrm{V}$ & 4.08 \\
\hline $054 \quad 7948903 \mathrm{~V}$ & 120.70 & $055 \quad 788 \quad 52 \quad 25 \mathrm{~V}$ & 3.91 \\
\hline 054_792_64_07_V & 95.24 & $044 \quad 789 \_89 \_44 \_\mathrm{V}$ & 3.81 \\
\hline 063_794_55_14_V & 89.05 & 055_788_49_31_V & 3.70 \\
\hline $\begin{array}{lllll}055 & 787 & 63 & 31 & \mathrm{~V}\end{array}$ & 88.58 & $06479265 \quad 45 \mathrm{~V}$ & 3.68 \\
\hline $0557870107 \mathrm{~V}$ & 87.96 & $055 \quad 788 \quad 74 \quad 37 \mathrm{~V}$ & 1.87 \\
\hline $064 \quad 795 \_02 \_44 \mathrm{~V}$ & 86.01 & $055 \quad 788 \_64 \quad 32 \_\mathrm{V}$ & 1.26 \\
\hline $064 \_795 \_54 \_86 \_\mathrm{V}$ & 82.98 & 055 788_45_57_V & 0.91 \\
\hline
\end{tabular}

Levando em consideração todo o conjunto de dados, observa-se que a maior parte das OAEs do Distrito Federal inspecionadas por meio do SIGOA-DF se encontram em baixo nível de deterioração (36.4\%), seguido dos níveis médio (35.1\%), alto (11.3\%), sofrível (4.8\%) e crítico (2.4\%). Estes dados se encontram visualmente na Figura 7.

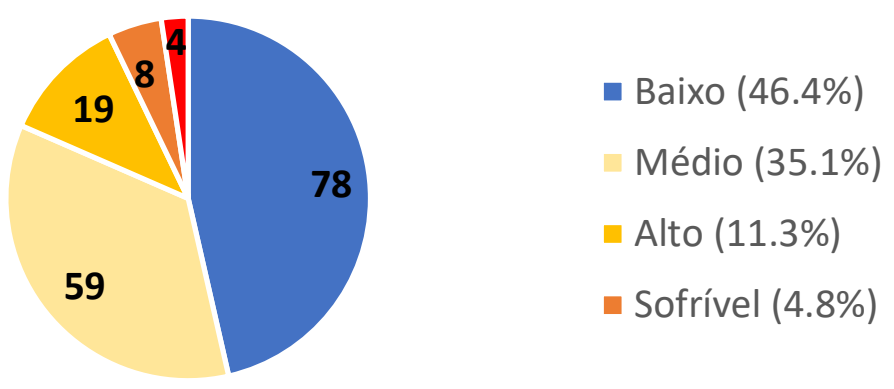

Figura 7- Níveis de Deterioração das OAEs analisadas 
Os dados obtidos podem ser distribuídos em um gráfico de dispersão, apresentado na Figura 8. Observa-se que, de fato, a maior parte das estruturas analisadas se encontram nos níveis baixo e médio, diminuindo a quantidade de OAEs à medida que se aumenta o nível de deterioração, e consequentemente os $\mathrm{G}_{\mathrm{d}}$ analisados.

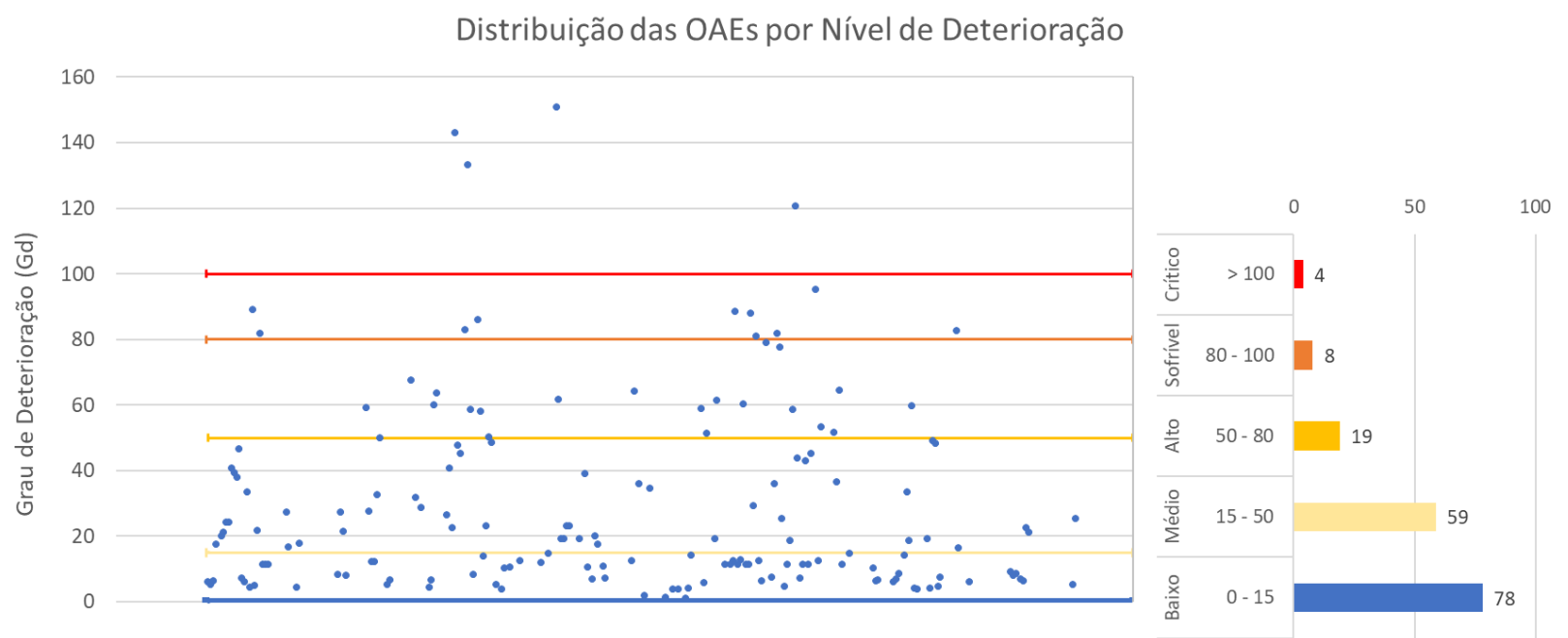

Figura 8 - Distribuição das OAEs por nível de Deterioração

A integração do SIGOA-DF a uma plataforma de georreferenciamento viabilizou a inserção dos pontos do banco de dados no mapa, sendo possível a caracterização dos pontos de acordo com o $\mathrm{G}_{\mathrm{d}}$ calculado. Parte do mapa gerado se encontra na Figura 9, onde observa-se grande concentração de OAEs com elevada deterioração na região central da cidade (Plano Piloto).
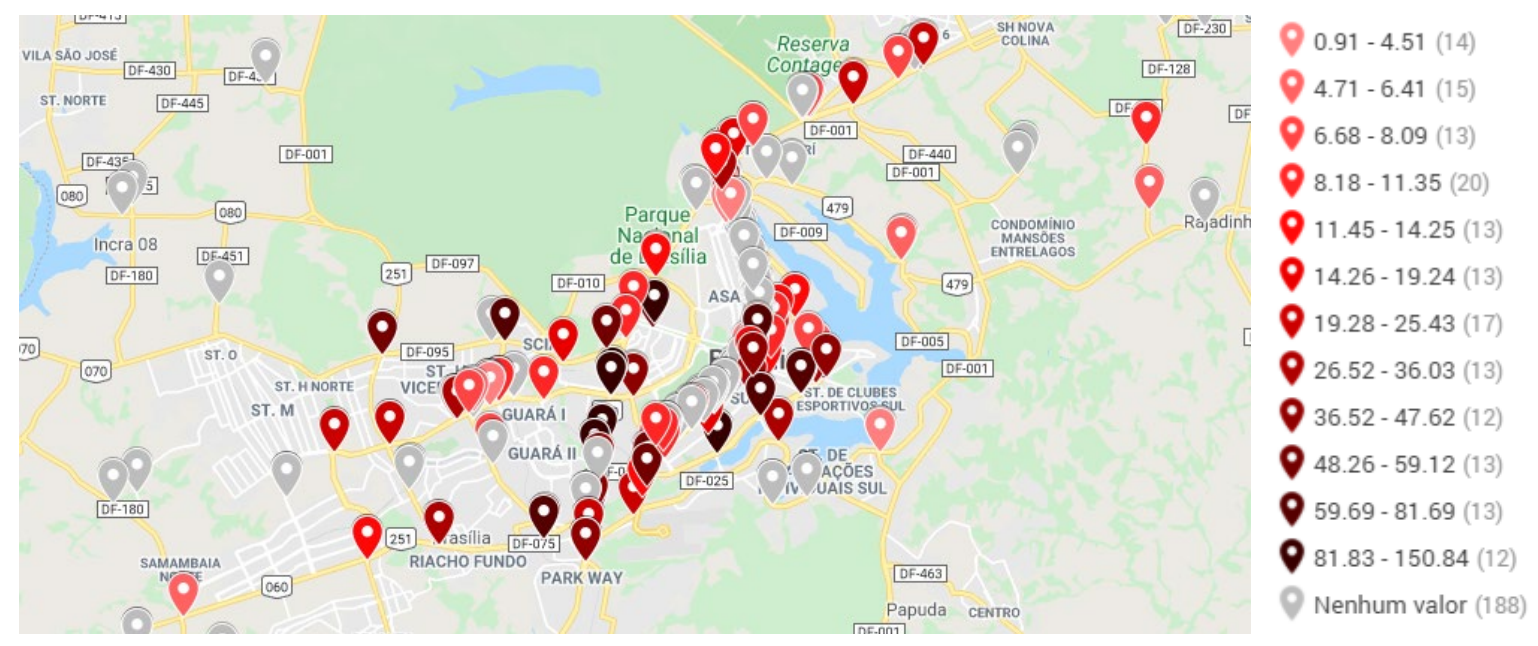

Figura 9 - Classificação das OAEs exportadas em plataforma de georreferenciamento

\subsection{Análise geral das OAEs inspecionadas}

Os dados coletados pela metodologia GDE/UnB utilizada na inspeção pela Ficha de Inspeção do SIGOA-DF permitem a visualização um panorama geral do grau de deterioração tanto dos elementos $\left(\mathrm{G}_{\mathrm{de}}\right)$ quanto das famílias que caracterizam cada um deles $\left(\mathrm{G}_{\mathrm{df}}\right)$. Para cada família, obtém-se de parâmetros como o Grau de Deterioração Médio da Família, o Grau de Deterioração 
Médio dos Elementos, bem como a quantidade de elementos que a compõem. A Tabela 3 mostra os parâmetros calculados para cada família inspecionada utilizando a Ficha de Inspeção.

Tabela 3 - Análise dos parâmetros das Famílias dos dados analisados

\begin{tabular}{|l|c|c|c|}
\hline \multicolumn{1}{|c|}{ Família } & Gdf Médio & $\begin{array}{c}\text { Gde Médio dos } \\
\text { Elementos }\end{array}$ & $\begin{array}{c}\text { Número Elementos } \\
\text { Inspecionados }\end{array}$ \\
\hline Cortina & 22.10 & 15.37 & 88 \\
\hline Cortina Central & 11.61 & 14.55 & 11 \\
\hline Guarda Rodas & 23.68 & 16.37 & 326 \\
\hline Longarina & 16.72 & 10.48 & 85 \\
\hline Pilar & 25.25 & 9.61 & 966 \\
\hline Tabuleiro & 36.51 & 18.49 & 555 \\
\hline Transversina & 18.20 & 8.04 & 76 \\
\hline
\end{tabular}

A partir da tabela, pode-se observar que a maior deterioração ocorre nos elementos dos tipos Tabuleiro, Pilar e Guarda-Rodas, em ordem decrescente. Averígua-se ainda que esses elementos, além de mais expostos ao fluxo de veículos no geral, foram os elementos mais inspecionados.

\subsection{Análise geral dos danos}

A caracterização dos danos, bem como a sua associação às tipologias ou famílias de elementos é de grande importância para um estudo mais aprofundado da deterioração das estruturas. Uma vez obtido o banco de dados com as informações detalhadas a respeito da recorrência de danos em cada elemento inspecionado, foi possível a sintetização dessas por meio da Ferramenta de Análise Geral presente no SIGOA-DF. A Tabela 4 apresenta os tipos de danos levados em consideração na metodologia GDE/UnB, assim como o número de ocorrências de cada um deles em elementos inspecionados e os Fatores de Intensidades $\left(\mathrm{F}_{\mathrm{i}}\right)$ Médios calculados.

Tabela 4 - Análise geral dos danos nas OAEs avaliadas

\begin{tabular}{|l|c|c|c|}
\hline Dano & Ocorrências & \% & Fi Médio \\
\hline Abrasão do concreto & 231 & $6.0 \%$ & 1.48 \\
\hline Cobrimento deficiente & 282 & $7.3 \%$ & 1.80 \\
\hline Corrosão de armaduras & 426 & $11.0 \%$ & 2.06 \\
\hline Desagregação & 237 & $6.1 \%$ & 1.92 \\
\hline Deslocamento por empuxo & 4 & $0.1 \%$ & 2.50 \\
\hline Desplacamento & 336 & $8.7 \%$ & 2.23 \\
\hline Desvio de geometria & 11 & $0.3 \%$ & 1.73 \\
\hline Eflorescência & 506 & $13.1 \%$ & 1.89 \\
\hline Falhas de concretagem & 368 & $9.5 \%$ & 1.15 \\
\hline Flechas & 2 & $0.1 \%$ & 2.00 \\
\hline Infiltração de água & 510 & $13.2 \%$ & 1.51 \\
\hline Manchas & 950 & $24.6 \%$ & 1.83 \\
\hline Sinais de Esmagamento & 0 & $0.0 \%$ & 0.00 \\
\hline
\end{tabular}

Por fim, o processamento dos dados realizado pelo SIGOA-DF possibilitou também a obtenção da recorrência dos danos específicos para cada família. Dessa maneira, pode ser feita uma análise das manifestações patológicas mais comuns em cada tipo de elemento, bem como de suas intensidades. 


\section{CONCLUSÕES}

O presente trabalho teve como objetivo principal o desenvolvimento de uma plataforma de gestão e inspeção de Obras de Arte Especiais baseada na metodologia do Grau de Deterioração de Estruturas desenvolvida pela Universidade de Brasília (GDE/UnB). Apesar de a metodologia ter sido inicialmente desenvolvida para o estudo do grau de deterioração de edifícios em geral, as adaptações feitas na literatura trouxeram novas formulações e modificações que permitiram o aprimoramento da avaliação, possibilitando a sua aplicação também a Obras de Arte Especiais.

A revisão bibliográfica possibilitou um panorama geral das atuais soluções utilizadas no Brasil e no mundo no que tange os sistemas de gerenciamento e procedimentos de inspeção de edificações, viadutos e pontes. Ademais, recentes acidentes na cidade de Brasília, como a queda de um viaduto na zona central no ano de 2018) alertaram para a importância do gerenciamento, manutenção e fiscalização do estado de conservação de edificações para garantir a segurança e usabilidade de seus usuários.

O desenvolvimento do SIGOA-DF contribuiu para uma maior facilidade e padronização na coleta de dados referentes a diversas pontes e viadutos do Distrito Federal por estudantes da Universidade de Brasília, cujos trabalhos se basearam na utilização da metodologia GDE/UnB, aliada à ferramenta de inspeção. $\mathrm{O}$ desenvolvimento e aprimoramento da plataforma em paralelo à coleta $\mathrm{e}$ subsequente processamento dos dados garantiu a criação de uma base de dados sólida para a testagem e ajuste da plataforma.

A partir dos dados coletados por Saraiva (2020), Chaves (2020) e Pereira (2020), além de outros estudantes do curso de engenharia civil da Universidade de Brasília, foi viabilizado o processamento preliminar de 168 OAEs, além de uma análise básica. Essa análise retratou importantes informações, como as Obras de Arte Especiais em estado mais crítico no Distrito Federal, bem como as patologias mais recorrentes encontradas e o grau de deterioração de elementos, famílias e estruturas globais. A criação do banco de dados possibilitou também a sintetização de informações valiosas, das quais muitas outras análises podem ser feitas.

\section{AGRADECIMENTOS}

À Universidade de Brasília, essencial no processo de formação e aprendizado do conteúdo e habilidades necessárias para a elaboração deste e outros trabalhos.

Aos professores, pelas correções e ensinamentos que permitiram a elaboração deste e outros trabalhos

À comissão organizadora do CONPAT 2021, pela oportunidade da submissão deste trabalho.

\section{REFERÊNCIAS}

Boldo, P. (2002), Análise Quantitativa de Estruturas de Concreto Armado de Edificações no Âmbito do Exército Brasileiro. Dissertação de Mestrado, Publicação E.DM-001A/02, Departamento de Engenharia Civil e Ambiental, Universidade de Brasília, Brasília, DF, 295 p.

Castro, E. K. (1994), Desenvolvimento de Metodologia para Manutenção de Estruturas de Concreto Armado. Dissertação de Mestrado, Publicação No: E.DM-004A/94, Departamento de Engenharia Civil, Universidade de Brasília, Brasília, DF, 155 p.

Chaves, L. (2020) Avaliação de Obras de Arte Especiais da cidade de Brasília pela Metodologia do Grau de Deterioração Estrutural da Universidade de Brasília. UnB 
Elbehairy, H. (2007), Bridge management system with Integrated Life Cycle Cost Optimization. 2007. 253-259 f. University of Waterloo

Euqueres, P. (2011); Metodologia de Inspeção em Estruturas de Pontes de Concreto Armado. Dissertação de Mestrado, Escola de Engenharia Civil, Universidade Federal de Goiás, Goiânia, $\mathrm{GO}, 168 \mathrm{p}$.

Fonseca, R. P. (2007); A Estrutura do Instituto Central de Ciências: Aspectos Históricos, Científicos e Tecnológicos de Projeto, Execução, Intervenções e Propostas de Manutenção. Dissertação de Mestrado em Estruturas e Construção Civil, Publicação E.DM - 006 A/07, Departamento de Engenharia Civil e Ambiental, Universidade de Brasília, Brasília, DF, 213p.

Klein, D., GASTAL, F., CAMPANOLO, J.L \& SILVA FILHO, L. C. (1991); “Critérios adotados na vistoria e avaliação de obras de arte", XXV Jornada Sul Americana de Engenharia Estrutural, Porto Alegre, pp.185-196, novembro.

Lopes, B. A. R. (1998); “Sistema de Manutenção Predial para Grades Estoques de Edifícios: Estudo para inclusão do componente "Estrutura de Concreto". Dissertação de Mestrado, Publicado No: E.DM-011A/98, Departamento de Engenharia Civil, Universidade de Brasília, Brasília, DF, 308p.

Mitre, M. P. (2005); Metodologia para inspeção e diagnóstico de pontes e viadutos de concreto / M. P. Mitre - São Paulo, 148p.

Moscoso, Y. F. M. (2017). Modelos de Degradação para Aplicação em Sistemas de Obras de Arte Especiais - OAEs. Tese de Doutorado em Estruturas e Construção Civil, Publicação E.TD3A/17, Departamento de Engenharia Civil e Ambiental, Universidade de Brasília, Brasília, DF, $185 \mathrm{p}$.

Pereira, M. S. (2020); Avaliação de Obras de Arte Especiais da cidade de Brasília (lote 1) pela Metodologia do Grau de Deterioração Estrutural da Universidade de Brasília. UnB

Saraiva, C. (2020); Avaliação de Obras de Arte Especiais da cidade de Brasília pela Metodologia do Grau de Deterioração Estrutural da Universidade de Brasília. UnB

Verly, R. C. (2015); Avaliação de Metodologias de Inspeção como Instrumento de Priorização de Intervenções em Obras de Arte Especiais. Dissertação de Mestrado em Estruturas e Construção Civil, Publicação E.DM-015A/15, Departamento de Engenharia Civil e Ambiental, Universidade de Brasília, Brasília, DF, 178p. 